\title{
Effect of antioxidant supplementation containing L-carnitine on semen parameters: a prospective interventional study
}

\author{
Leila Nazari ${ }^{1}$, Saghar Salehpour ${ }^{1}$, Sedighe Hosseini ${ }^{1}$, Farzad Allameh ${ }^{2}$, Ferdoos Jahanmardi ${ }^{1}$, Elham Azizi ${ }^{3}$, \\ Robabeh Ghodssi-Ghassemabadi ${ }^{4}$, Teibeh Hashemi ${ }^{1}$
}

\author{
${ }^{1}$ Department of Obstetrics and Gynecology, Preventative Gynecology Research Center, Shahid Beheshti \\ University of Medical Sciences, Tehran, Iran \\ 2Department of Urology, Preventative Gynecology Research Center, Shahid Beheshti University o f Medical \\ Sciences, Tehran, Iran \\ ${ }^{3}$ Department of Biology and Anatomical Sciences, Student Research Committee, Shahid Beheshti University of \\ Medical Sciences, Tehran, Iran \\ ${ }^{4}$ Department of Biostatistics, Faculty of Medical Sciences, Tarbiat Modares University, Tehran, Iran
}

\begin{abstract}
Objective: One of the remarkable causes of infertility in men is oxidative stress having a reducing effect on their reproductive function. In the present study, we investigated the efficacy of supplementation with antioxidants and L-Carnitine (contained in Androferti) on semen parameters.

Methods: We included 180 infertile male patients diagnosed with idiopathic oligoastenoteratozoospermia (OAT) in this study, and we analyzed the semen sample from 59 patients before and after oral antioxidant treatment, with the commercial name of Androferti (containing $1500 \mathrm{mg}$ of L-Carnitine, $60 \mathrm{mg}$ of vitamin C, $20 \mathrm{mg}$ of coenzyme Q10, $10 \mathrm{mg}$ of vitamin $\mathrm{E}, 10 \mathrm{mg}$ of zinc, $200 \mu \mathrm{g}$ of vitamin B9, $50 \mu \mathrm{g}$ of selenium, $1 \mu \mathrm{g}$ of vitamin B12). All of the patients received Androferti twice a day for 3 months.

Results: There were significant improvements in the sperm concentration $(p=0.004)$ after the antioxidant supplementation. There was also a meaningfully improvement in sperm morphology $(p=0.01)$ after treatment. However, sperm motility was not significantly altered after antioxidant treatment $(p=0.2)$.

Conclusions: Antioxidants supplementation containing $1500 \mathrm{mg} \mathrm{L}$-carnitine can improve the semen quality in infertile men diagnosed with idiopathic OAT. However, further studies are required to determine the antioxidant effects on reproduction function.
\end{abstract}

Keywords: antioxidant supplementation, semen analysis, oxidative stress, reactive oxygen species (ROS)

\section{INTRODUCTION}

Infertility is a global problem that affects about $15 \%$ of couples intending to conceive (Practice Committee of American Society for Reproductive Medicine, 2013). The World Health Organization (WHO) defines infertility as the inability of couples to achieve a successful pregnancy after 12 months of regular intercourse using no contraception (Practice Committee of American Society for Reproductive Medicine, 2013). Male factor infertility is one of the common causes of pregnancy failure in infertile couples. Idiopathic male infertility, which involves the majority of infertile men, affects the semen quality without an identifiable cause. The improvement in semen quality is still a challenge in the treatment of these men (Agarwal et al., 2019).
Reactive oxygen species (ROS) have been proposed as fundamental factors that may negatively affect semen quality (Tremellen, 2008). ROS are produced as the end products when a cell uses oxygen, and their increased levels can lead to cellular damage (Tremellen, 2008). Every human body is naturally equipped with the ability to neutralize the excess ROS, which can be produced either due to endogenous or exogenous factors (Smits et al., 2018). Any disturbance in the balance between ROS and antioxidants can inevitably lead to oxidative stress. Oxidative stress is defined as the imbalance between the ROS production and the body's ability to detoxify them and a considerable contributor to the pathophysiology of male infertility (Behrman et al., 2001). Several exogenous factors (e.g., environmental pollutions, smoking, alcohol consumption, and poor nutrition) and endogenous factors (e.g., obesity, infections, and chronic and autoimmune diseases) are mentioned in the literature as the most common causes of oxidative stress in the reproductive tract (Tremellen, 2008). Oxidative stress has been reported in most of the infertile men. Although a physiological level of ROS is essential for sperm maturation, capacitation, hyper-activation, and fertilization, oxidative stress can lead to detrimental effects on spermatogenesis and semen quality (Rajesh Kumar et al., 2002; Guthrie \& Welch, 2012). Antioxidants are agents that prevent oxidative damage to cells and tissues via directly scavenging and inactivating excessive ROS and repairing its damages (Mirończuk-Chodakowska et al., 2018). Some of the wellknown enzymatic and non-enzymatic agents which act as antioxidants in the human body are catalase, glutathione reductase, superoxide dismutase, glutathione peroxidase, ascorbic acid, alpha-tocopherol, ferritin, and transferrin (Gupta et al., 2007).

L-carnitine is one of the natural antioxidants existing in - namely - the seminal plasma of mammals, that inactivate ROS, inhibit lipid peroxidation, and protect the sperm membrane (Gülçin, 2006; Ahmed et al., 2017). This antioxidant is mainly secreted from mammalian epithelium into the epididymal plasma, and it finally builds up in the sperm cells and protects them from oxidant injury (Ahmed et al., 2017). Moreover, L-carnitine is an essential co-factor for beta-oxidation, which leads to energy production from lipids in the mitochondria (Ahmed et al., 2017). Additionally, L-carnitine is an inexpensive natural antioxidant, and almost free of side effects (DiNicolantonio et al., 2019). Hence, L-carnitine supplementation has been proposed to 
be useful for the treatment of infertile men (Agarwal et al., 2004; Balercia et al., 2005; Garolla et al., 2005; Cavallini et al., 2004; Lenzi et al., 2004). However, the efficacy of L-carnitine treatment in patients diagnosed with idiopathic oligoastenoteratozoospermia (OAT) is still under debate. The present study aimed to assess the influence of an antioxidant-containing supplementation (containing $1500 \mathrm{mg}$ of L-Carnitine, $60 \mathrm{mg}$ of vitamin C, $20 \mathrm{mg}$ of coenzyme Q10, $10 \mathrm{mg}$ of vitamin $\mathrm{E}, 10 \mathrm{mg}$ of zinc, $200 \mu \mathrm{g}$ of vitamin $\mathrm{B} 9,50 \mu \mathrm{g}$ of selenium, $1 \mu \mathrm{g}$ of vitamin B12) on the semen parameters of infertile men with idiopathic OAT.

\section{MATERIALS AND METHODS}

\section{Study population}

In the present prospective interventional study, we recruited 180 infertile men between 20-45 years of age, diagnosed with idiopathic OAT from Taleghani Hospital in Tehran, from 2017 to 2018. We included 70 patients in the study, according to the Gehan's formula. We obtained two semen samples from each patient: one sample before starting the antioxidant treatment and another sample after completing the antioxidant treatment. We took 11 patients off the study, because they did not return for the second analysis after the treatment (Figure 1). The Ethics Committee of the Shahid Beheshti University of Medical Sciences approved the study (IR.SBMU. RETECH.REC.1397.1359), and the trial received number IRCT20190426043379N1. All the participants signed a written informed consent before entering the study.

\section{Inclusion criteria}

All the patients who received the treatment had more than one year of unprotected intercourse. The patients included in our study had at least one abnormal semen parameter (sperm concentration less than $15 \times 10^{6}$ per $\mathrm{ml}$, sperm motility less than $40 \%$, and normal morphology less than $4 \%$ ), according to the WHO criteria (World Health Organization, 2010). All the participants had less than 45 years of age, a BMI under 30, did not smoke, and had no addiction. An expert urologist visited all of them.

\section{Exclusion criteria}

The exclusion criteria were azoospermia, prostatitis, genital trauma, testicular torsion, any genital disease (e.g., cryptorchidism, current genital inflammation, varicocele), endocrinopathy, any history of epididymo-orchitis, genital surgery, urinary tract infection, severe general or central nervous system disease, $Y$ chromosome microdeletions or karyotype abnormalities, and recent sexually transmitted disease. In addition, we excluded the patients if they had any previous hormonal therapy or if they consumed cytotoxic drugs, alcohol drinking behaviors, drug abuse, immunosuppressants, anticonvulsive, androgens, antiandrogens, or any psychological or physiological abnormalities, which could impair their reproduction function or spermatogenesis. Also, having any diseases of the liver and biliary system, significant poor function of the kidneys, occupational and environmental exposures to potential reproductive toxins, and intolerance to the drug were other excluding factors.

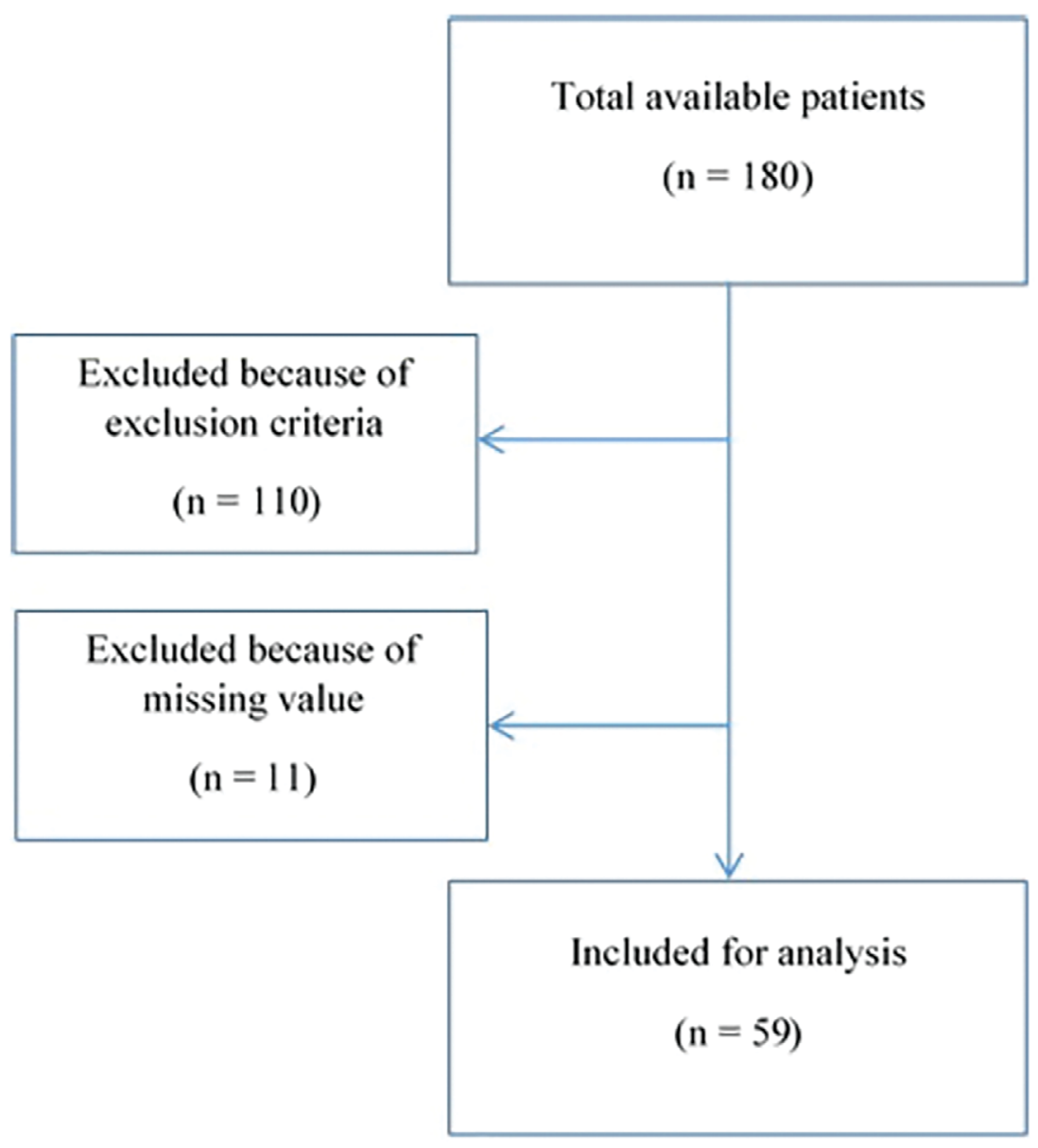

Figure 1. Flowchart of the study population 


\section{Study design}

Before starting the treatment, a semen sample was collected from each patient after 3-5 days of abstinence. The samples were incubated for $20-30 \mathrm{~min}$ at $37^{\circ} \mathrm{C}$ to be liquefied. After sample liquefaction, the semen parameters were determined according to WHO criteria (World Health Organization, 2010). For the antioxidant treatment, all patients received the Androferti supplement (Daru Darman Parmida, Iran) orally and twice daily. For detecting any effect of treatment on the semen parameters, the treatment continued for 3 months, because every spermatogenesis cycle period lasted for at least 3 months. The other semen sample was collected from each patient after the antioxidant supplementation treatment and analyzed together with the pre-treatment. Tolerability was evaluated based on the probable adverse effects reported by the patients and the physical examination conducted during the patient visit.

\section{Statistical analysis}

We used the mean, median, standard deviation (SD), and interquartile range (IQR) to report the quantitative data. The qualitative data was represented using frequency and percentage. We used the non-parametric Wilcoxon signed-ranks test to compare data before and after treatment, with $p<0.05$ considered to be statistically significant. All the above-mentioned statistical analyses were carried out in SPSS software version 24.

\section{RESULTS}

We had 59 infertile men, with at least one year of infertility accomplishing the treatment course. We analyzed the patients' semen parameters and compared them based on pre-and post-antioxidant treatment. The patients had mean age of $34.5 \pm 4.16$ (range: $26-40$ ). Among all patients, $20.3 \%$ were in the age range of $26-30$ years, $35.6 \%$ were between $31-35$ years, and $44.1 \%$ were between $36-$ 40 years of age. There was $100 \%$ treatment compliance among the patients. The mean marital age among the study subjects was $7.5 \pm 5.1$ years (range: $1-21$ years). Out of 59 patients, $11.9 \%$ had normal weight, $1.7 \%$ were underweight, and $84.6 \%$ were overweight. Table 1 depicts the baseline characteristics of the patients.

Table 2 shows that the concentration of sperm increased significantly after the antioxidants treatment $(2.5$ $\times 10^{7}\left[1.2 \times 10^{7}-5 \times 10^{7}\right]$ vs. $3.6 \times 10^{7}\left[1.5 \times 10^{7}-7 \times\right.$ $\left.10^{7}\right], p=0.004$ ) (Table 2 ). In addition, sperm morphology markedly increased after the treatment (1\% [0\% - 2\%] vs. $1 \%[0 \%-3 \%], p=0.013$ ) (Table 2 ), although the increased value of sperm motility after treatment was not statistically significant. (28\% [10\% - 50\%] vs. 35\% [12\% - 50\%], $p=0.2$ ) (Table 2).

\section{DISCUSSION}

Recent reports have indicated that semen quality has declined notably during the past 20 years (Alahmar, 2018), for which several causes have been discussed. Environmental factors (e.g., metal toxicity, chemicals, and other pollutants, radiation, heat, etc.), as well as obesity, inflammation, smoking, and ROS exposure, are counted as some causes of decreased spermatogenesis and sperm DNA integrity (Darbandi et al., 2018). Sperm quality and quantity - the determinative factors for male fertility - can be impacted by excessive ROS in the reproductive tract and semen. Therefore, sufficient antioxidant levels should be steadily maintained in the body to prevent excessive ROS from impairing spermatozoa proteins, lipid membranes, and DNA integrity. The present study demonstrates that antioxidants supplement containing $1500 \mathrm{mg} \mathrm{L}$-carnitine can improve semen quality.

Previously, several supplements, including vitamins C and E, L-carnitine, coenzyme Q10, pentoxifylline, and trace elements (such as zinc and selenium), have been investigated for their conservative actions against ROS, individually or in combination. Accordingly, some of these antioxidant supplements have positively influenced sperm quality and quantity in the semen samples of participants, the DNA integrity of sperms, the total antioxidant capacity of seminal plasma, the success rate of pregnancy, and the in vitro fertilization outcomes (Agarwal et al., 2004; Balercia et al., 2005; Cavallini et al., 2004; Lenzi et al., 2004; Lombardo et al., 2011; Rolf et al., 1999; Comhaire et al., 2000; Paradiso Galatioto et al., 2008; Hargreave et al., 1984; Vicari \& Calogero, 2001; Vicari et al., 2002; Lenzi et al., 2003). In addition, there are reports of synergistically boosted positive effects from multi-therapy trials with antioxidants. However, the exact mechanism of the antioxidants' role in conserving the sperm concentration has not yet been identified, the suppression of ROS-induced sperm damage has been repeatedly suggested as a probable mechanism (Agarwal et al., 2004; Lombardo et al., 2011).

The present study showed that treatment with an edible antioxidant supplement (containing $1500 \mathrm{mg}$ of L-Carnitine, $60 \mathrm{mg}$ of vitamin C, $20 \mathrm{mg}$ of coenzyme Q10, 10 $\mathrm{mg}$ of vitamin $\mathrm{E}, 10 \mathrm{mg}$ of zinc, $200 \mu \mathrm{g}$ of vitamin B9, 50 $\mu \mathrm{g}$ of selenium, $1 \mu \mathrm{g}$ of vitamin B12) for 3 months could

Table 1. Baseline characteristics of patients

\begin{tabular}{|l|l|l|l|}
\hline Variable & Category & Frequency & Percent \\
\hline Age (years) & $26-30$ & 12 & 20.3 \\
& $31-35$ & 21 & 35.6 \\
& $36-40$ & 26 & 44.1 \\
\hline Job & Self-employed & 45 & 76.3 \\
& Governmental & 14 & 23.7 \\
\hline Education level & Illiterate & 2 & 3.4 \\
& Undergraduate & 39 & 82.8 \\
\hline Body mass index $\left(\mathbf{k g} / \mathbf{m}^{\mathbf{2}}\right.$ ) & Academic degree & 14 & 13.8 \\
\hline & Normal & 1 & 1.7 \\
& overweight & 7 & 11.9 \\
\hline Marriage age (years) & $\leq 5$ & 51 & 86.4 \\
\hline & $6-10$ & 24 & 40.7 \\
& $11-15$ & 20 & 33.9 \\
\hline
\end{tabular}


Table 2. Description and comparison of semen parameters before and after treatment

\begin{tabular}{|l|c|c|c|c|c|}
\hline \multirow{2}{*}{ Semen parameter } & \multicolumn{2}{|c|}{ Before treatment } & \multicolumn{2}{c|}{ After treatment } & \multirow{2}{*}{ p-value } \\
\cline { 2 - 6 } & Median & IQR & Median & IQR & \\
\hline Concentration (sperm / mL) & $2.5 \times 10^{7}$ & $1.2 \times 10^{7}-5 \times 10^{7}$ & $3.6 \times 10^{7}$ & $1.5 \times 10^{7}-7 \times 10^{7}$ & 0.004 \\
\hline Morphology & $1 \%$ & $0 \%-2 \%$ & $1 \%$ & $0 \%-3 \%$ & 0.01 \\
\hline Motility & $28 \%$ & $10 \%-50 \%$ & $35 \%$ & $12 \%-50 \%$ & 0.2 \\
\hline
\end{tabular}

improve sperm concentration and morphology in infertile men with idiopathic AOT. Previously, in a study by Lenzi et al. (2004), they showed that a combined L-carnitine (2 $\mathrm{g} / \mathrm{d}$ ) and L-acetyl-carnitine $(1 \mathrm{~g} / \mathrm{d})$ treatment for 2 months in infertile males with OAT increased sperm motility, but the other semen parameters did not improve in that study. In contrast to their findings, our study showed that antioxidant supplements containing L-carnitine $(1500 \mathrm{mg})$ for 3 months could improve sperm concentration and morphology in men with idiopathic OAT, but not the sperm motility. In another study, oral antioxidant treatment containing $1500 \mathrm{mg}$ of L-carnitine for 3 months increased sperm concentration, motility, and morphology in infertile patients with "known" OAT, which was not found in our study (Abad et al., 2013).

Nevertheless, there are also studies reporting no impact on semen parameters caused by antioxidants (Agarwal et al., 2004; Lombardo et al., 2011). Comparing the compatible and opposite outcomes of various studies is challenging for several reasons. One reason is that many of such studies are randomized placebo-controlled trials or case-control studies, while others are performed under open, uncontrolled, or prospective methods. Antioxidants have also been studied in various doses and for varying durations, from 3 to 6 months, in various number of patients with idiopathic or known OAT. Another reason is that the negative results obtained from some similar studies can be interpreted as not enough reliable because of their small population size, insufficient doses of antioxidants, and/or short treatment duration (Agarwal et al., 2004). The root of antioxidant supplement administration also contributes to its effectiveness on the seminal fluid parameters, such that some studies report no appreciable improvement in sperm parameters by the oral administration of antioxidants (Agarwal et al., 2004; Lombardo et al., 2011).

Regarding all the above-mentioned challenges and considering the reports that confirm the positive effects of antioxidant supplementation on sperm parameters, including the present one, we conclude that the dose and duration of treatment with antioxidants require being optimized in future related studies. We must also determine the seminal parameters that specifically benefit from a particular antioxidant and the efficacy of that specific antioxidative agent in mono- or poly-therapy. Also, adhering to a supplement treatment duration of at least 3 months, especially in cases of severe oxidative stress exposure, is an essential factor to be considered in studies since spermatozoa maturation takes around 72 days (Alahmar, 2018).

\section{CONCLUSIONS}

According to the findings of the present study, a treatment intervention with antioxidant supplementation containing $1500 \mathrm{mg}$ L-carnitine via oral administration can improve two sperm parameters in semen samples, including sperm concentration and morphology, while it did not change the sperm motility. Considering the demonstrated safety profile of L-carnitine supplementation, the present findings corroborate the oral supplementation with L-carnitine as a partially efficient intervention to improve the fertility in the idiopathic OAT male patients owing to its antioxidant potentials.

\section{ACKNOWLEDGMENTS}

The present article was financially supported by the Research Department of the School of Medicine, Shahid Beheshti University of Medical Sciences. We especially thank Maryam Karimi for her great help and support in performing this study.

\section{CONFLICT OF INTEREST}

None.

\section{Corresponding author:}

Teibeh Hashemi

Department of Obstetrics and Gynecology

IVF Center, Taleghani Hospital

Tehran, Iran.

E-mail: hashemit89@yahoo.com

\section{REFERENCES}

Abad C, Amengual MJ, Gosálvez J, Coward K, Hannaoui N, Benet J, García-Peiró A, Prats J. Effects of oral antioxidant treatment upon the dynamics of human sperm DNA fragmentation and subpopulations of sperm with highly degraded DNA. Andrologia. 2013;45:211-6. PMID: 22943406 DOI: 10.1111/and.12003

Agarwal A, Nallella KP, Allamaneni SS, Said TM. Role of antioxidants in treatment of male infertility: an overview of the literature. Reprod Biomed Online. 2004;8:616-27. PMID: 15169573 DOI: 10.1016/S1472-6483(10)61641-0

Agarwal A, Parekh N, Panner Selvam MK, Henkel R, Shah R, Homa ST, Ramasamy R, Ko E, Tremellen K, Esteves S, Majzoub A, Alvarez JG, Gardner DK, Jayasena CN, Ramsay JW, Cho CL, Saleh R, Sakkas D, Hotaling JM, Lundy SD, et al. Male Oxidative Stress Infertility (MOSI): Proposed Terminology and Clinical Practice Guidelines for Management of Idiopathic Male Infertility. World J Mens Health. 2019; 37:296-312. PMID: 31081299 DOI: 10.5534/ wjmh. 190055

Ahmed SH, Ahsan S, Iqbal T, Burney SA. Relationship of seminal free L-Carnitine with functional spermatozoal characteristics: results from an observational study conducted in a tertiary care hospital of Karachi, Pakistan. J Pak Med Assoc. 2017;67:280-4. PMID: 28138186

Alahmar AT. The effects of oral antioxidants on the semen of men with idiopathic oligoasthenoteratozoospermia. Clin Exp Reprod Med. 2018;45:57-66. PMID: 29984205 DOI: 10.5653/cerm.2018.45.2.57 
Balercia G, Regoli F, Armeni T, Koverech A, Mantero F, Boscaro $M$. Placebo-controlled double-blind randomized trial on the use of L-carnitine, L-acetylcarnitine, or combined L-carnitine and L-acetylcarnitine in men with idiopathic asthenozoospermia. Fertil Steril. 2005;84:662-71. PMID: 16169400 DOI: $10.1016 /$ j.fertnstert.2005.03.064

Behrman HR, Kodaman PH, Preston SL, Gao S. Oxidative stress and the ovary. J Soc Gynecol Investig. 2001;8:S402. PMID: 11223371 DOI: $10.1177 / 1071557601008001 S 13$

Cavallini G, Ferraretti AP, Gianaroli L, Biagiotti G, Vitali G. Cinnoxicam and L-carnitine/acetyl-L-carnitine treatment for idiopathic and varicocele-associated oligoasthenospermia. J Androl. 2004;25:761-70. PMID: 15292108 DOI: 10.1002/j.1939-4640.2004.tb02853.x

Comhaire FH, Christophe AB, Zalata AA, Dhooge WS, Mahmoud AM, Depuydt CE. The effects of combined conventional treatment, oral antioxidants and essential fatty acids on sperm biology in subfertile men. Prostaglandins Leukot Essent Fatty Acids. 2000;63:159-65. PMID: 10991774 DOI: $10.1054 /$ plef.2000.0174

Darbandi M, Darbandi S, Agarwal A, Sengupta P, Durairajanayagam D, Henkel R, Sadeghi MR. Reactive oxygen species and male reproductive hormones. Reprod Biol Endocrinol. 2018;16:87. PMID: 30205828 DOI: 10.1186/ s12958-018-0406-2

DiNicolantonio JJ, Niazi AK, McCarty MF, Lavie CJ, Liberopoulos $\mathrm{E}$, O'Keefe $\mathrm{JH}$. L-carnitine for the treatment of acute myocardial infarction. Rev Cardiovasc Med. 2019;15:5262. PMID: 24762466

Garolla A, Maiorino M, Roverato A, Roveri A, Ursini F, Foresta $C$. Oral carnitine supplementation increases sperm motility in asthenozoospermic men with normal sperm phospholipid hydroperoxide glutathione peroxidase levels. Fertil Steril. 2005;83:355-61. PMID: 15705374 DOI: 10.1016/j.fertnstert.2004.10.010

Gülçin I. Antioxidant and antiradical activities of L-carnitine. Life Sci. 2006;78:803-11. PMID: 16253281 DOI: $10.1016 /$ j.Ifs.2005.05.103

Gupta S, Agarwal A, Banerjee J, Alvarez JG. The role of oxidative stress in spontaneous abortion and recurrent pregnancy loss: a systematic review. Obstet Gynecol Surv. 2007;62:335-47. PMID: 17425812 DOI: $10.1097 / 01$. ogx.0000261644.89300.df

Guthrie H, Welch $\mathrm{G}$. Effects of reactive oxygen species on sperm function. Theriogenology. 2012;78:1700-8. PMID: 22704396 DOI: $10.1016 /$ j.theriogenology.2012.05.002

Hargreave TB, Kyle KF, Baxby K, Rogers AC, Scott R, Tolley DA, Abel BJ, Orr PS, Elton RA. Randomised trial of mesterolone versus vitamin $C$ for male infertility. Scottish Infertility Group. Br J Urol. 1984;56:740-4. PMID: 6398725 DOI: 10.1111/j.1464-410X.1984.tb06160.x

Lenzi A, Lombardo F, Sgrò P, Salacone P, Caponecchia L, Dondero F, Gandini L. Use of carnitine therapy in selected cases of male factor infertility: a double-blind crossover trial. Fertil Steril. 2003;79:292-300. PMID: 12568837 DOI: $10.1016 / \mathrm{S} 0015-0282(02) 04679-4$
Lenzi A, Sgrò $P$, Salacone P, Paoli D, Gilio B, Lombardo F, Santulli M, Agarwal A, Gandini L. A placebo-controlled double-blind randomized trial of the use of combined l-carnitine and I-acetyl-carnitine treatment in men with asthenozoospermia. Fertil Steril. 2004;81:1578-84. PMID: 15193480 DOI: $10.1016 /$ j.fertnstert.2003.10.034

Lombardo F, Sansone A, Romanelli F, Paoli D, Gandini L, Lenzi $A$. The role of antioxidant therapy in the treatment of male infertility: an overview. Asian J Androl. 2011;13:6907. PMID: 21685925 DOI: $10.1038 / a j a .2010 .183$

Mirończuk-Chodakowska I, Witkowska AM, Zujko ME. Endogenous non-enzymatic antioxidants in the human body. Adv Med Sci. 2018;63:68-78. PMID: 28822266 DOI: 10.1016/j.advms.2017.05.005

Paradiso Galatioto G, Gravina GL, Angelozzi G, Sacchetti A, Innominato PF, Pace G, Ranieri G, Vicentini C. May antioxidant therapy improve sperm parameters of men with persistent oligospermia after retrograde embolization for varicocele? World J Urol. 2008;26:97-102. PMID: 17982752 DOI: $10.1007 / \mathrm{s} 00345-007-0218-z$

Practice Committee of American Society for Reproductive Medicine. Definitions of infertility and recurrent pregnancy loss: a committee opinion. Fertil Steril. 2013;99:63. PMID: 23095139 DOI: 10.1016/j.fertnstert.2012.09.023

Rajesh Kumar T, Doreswamy K, Shrilatha B, Muralidhara. Oxidative stress associated DNA damage in testis of mice: induction of abnormal sperms and effects on fertility. Mutat Res. 2002;513:103-11. PMID: 11719095 DOI: 10.1016/ S1383-5718(01)00300-X

Rolf C, Cooper T, Yeung C, Nieschlag E. Antioxidant treatment of patients with asthenozoospermia or moderate oligoasthenozoospermia with high-dose vitamin $C$ and vitamin E: a randomized, placebo-controlled, double-blind study. Hum Reprod. 1999;14:1028-33. PMID: 10221237 DOI: $10.1093 /$ humrep/14.4.1028

Smits RM, Mackenzie-Proctor R, Fleischer K, Showell MG. Antioxidants in fertility: impact on male and female reproductive outcomes. Fertil Steril. 2018;110:578-80. PMID: 30196940 DOI: $10.1016 /$ j.fertnstert.2018.05.028

Tremellen K. Oxidative stress and male infertility--a clinical perspective. Hum Reprod Update. 2008; 14:243-58. PMID: 18281241 DOI: $10.1093 /$ humupd/dmn004

Vicari E, Calogero AE. Effects of treatment with carnitines in infertile patients with prostato-vesiculo-epididymitis. Hum Reprod. 2001;16:2338-42. PMID: 11679516 DOI: 10.1093/humrep/16.11.2338

Vicari E, La Vignera S, Calogero AE. Antioxidant treatment with carnitines is effective in infertile patients with prostatovesiculoepididymitis and elevated seminal leukocyte concentrations after treatment with nonsteroidal anti-inflammatory compounds. Fertil Steril. 2002;78:1203-8. PMID: 12477513 DOI: 10.1016/S0015-0282(02)04350-9

World Health Organization (WHO). WHO laboratory manual for the examination and processing of human semen. Geneva: World Health Organization; 2010. 